\title{
Antecedent Influence Factor Analysis on the Application of Cloud-computing-based Human Resource Management System
}

\author{
Chao Qin ${ }^{12, a^{*}}$ and Jun Chen ${ }^{2, a}$ \\ ${ }^{1}$ School of Education, Yunnan Minzu University, Kunming Yunnan, China \\ ${ }^{2}$ School of Business and Tourism, Yunnan University, Kunming Yunnan, China \\ aangelarqin@gmail.com
}

Keywords: Influence factors; Cloud compute; Human resource management system; Technology acceptance model

\begin{abstract}
With gradual deepening of cloud computing application in enterprise informatization construction, the human resources management system will also enter the cloud era. However, for the popularization and application of cloud-computing-based human resource management system, there are still many obstacles and influencing factors. Upon referencing technology acceptance model, this paper construct the acceptance model of cloud-computing-based human resource management system. The model has divided the antecedent influence factors which affect enterprise acceptance cloud computing human resources management system into three major factors including external environment, organizational characteristics and user individual characteristics, and in-depth dissected each factor impact on enterprise perceived usefulness and perceived ease of use
\end{abstract}

\section{Introduction}

With the universal application of computer technology in enterprise management, after human resources management system (HRMS) has entered the fourth generation - e human resource management system age[1], which e not only refers to "electronic", but owns the meaning of "efficiency" after going through salary computing systems age, salary / personnel management system age and human resources management system age. At the same time, a new generation of large-scale Internet applications in the Internet world urge the emergence of "cloud computing", a variety of cloud-computing-based applications begin to gradually extend to the enterprise informatization management and construction, especially in human resources management field, thus human resources management system ushers the cloud era.

"Cloud computing" is a sort of computing mode conception proposed in 2006 to use the Internet to achieve visiting sharing resources (such as facilities, storage devices, applications, etc.) as required, conveniently anytime and anywhere, and shields data center management, large-scale data processing, application program deployment. It can make enterprise users quickly apply or release resources according to their business, and pay for the using resources in the form of paying as required, meanwhile reduce the cost of operation and maintenance during providing qualified service [2].

The integration of cloud computing and human resources management system is believed to be able to reduce the operating cost and management cost of enterprise human resources management, improve the efficiency of enterprise human resources management, help enterprises get rid of scale and capital constraints. Therefore, cloud-computing-based human resource management system has the advantage of differentiation, high efficiency and low cost [3-4]. The application research and practice of cloud computing in the field of human resource management gradually bring an upsurge, human resources management software company will timely bring out products based on cloud computing and actively publicize and recommend enterprise users to migrate human resource management system into the cloud.gs, below). 


\section{Literature Review}

The research on cloud computing in China began in 2007, which has included 12181 pieces of various papers, periodicals and other relevant information. However, the research on human resource management under cloud computing environment is still in its initial stage, and the research field presents obvious imbalance status. Taking the six modules of human resource management including human resource planning, recruitment and allocation, training and development, performance management, salary welfare management and employee relationship management as example, existing research results are more concentrated in model building [5], cloud recruitment[6], and cloud training of cloud-computing-based human resources management system[7], while the performance management and salary welfare management and employee relationship management are relatively rare. In addition, literature research shows that the academic circles has also conducted many research and analysis on cloud-computing-based human resource management system for small and micro enterprises [8].

In the industry, at the beginning of 2012 , Oracle spent $\$ 1.9$ billion to purchase Taleo, a provider of cloud computing talent management software. And as early as the end of 2011, SAP announced using $\$ 3.4$ billion to purchase Success Factors, a provider of human resources software. Both technology giants have begun to get involved in the field of cloud computing human resource management system. Another large independent software company in the field of human resources management, workday also listed on the New York Stock Exchange on 31stOctober, 2012, marking the technology giant begin to actively layout the field of cloud-computing-based human resource management.

In China, based on cloud concept, Dayee, Hirede, and Beisen have in succession brought out cloud recruitment platform, able to adapt the changing demand of enterprise, dynamic and convenient network configuration resources, forming a relatively complete series of product lines including recruitment demand management, resume screening, talent assessment and dynamic talent pool etc. [9]. At the same time, cloud computing has become the leading technology of developing e-learning platform in enterprise training, and the research emphasis of HRE-learning, such as "E-learning" SaaS platform developed by CAS (Chinese Academy of Sciences)computer network information center "Public training learning platform for electronic and information industrial clusters cloud services" has been established in Dongguan electronic information enterprises, becoming indispensable training platform for local small and medium-sized enterprises[10] .

\section{Cloud-Computing-Based Human Resource Management System Acceptance Model}

Cloud-computing-based human resources management system has migrate organization internal isolated human resource management systems into the cloud, it is brand new and challenging application and system for traditional human resource administrator and the organization using cloud computing. It will greatly affect the application and popularization of cloud computing in the field of human resources management, whether these administrators and organization are willing to accept this brand new change. Therefore, with the help of referencing Technology Acceptance Model, construct cloud-computing-based Technology Acceptance Model, and analyze the influence factors that enterprise organization and human resource administrator accept cloud-computing-based human resource management system

Technology Acceptance Model. Technology Acceptance Model was proposed in 1989 by Davis[11], using rational behavior theory to study users' accepting information system, the initial proposal of this model is to explain the decisive factors for widely accepting computer. Technology Acceptance Model proposes that there are two main determining factors for accepting technology: (1)Perceived usefulness, this factor reflects individual opinion about the extent of improving and raising work performance in using a specific system; (2)Perceived ease of use, this factor reflects individual opinion about the easiness degree of using a specific system.

Technology acceptance model thinks that, the use of information system is determined by behavioral intention, and behavior intention is jointly determined by the attitude desiring to use and 
perceived usefulness; the attitude want to use is jointly determined by perceived usefulness and perceived ease of use; perceived usefulness is jointly determined by perceived ease of use and external variables; while external variables will also influence perceived usefulness and perceived ease of use. External variables include a variety of external factors, such as the characteristics of system design, user characteristics, task characteristics, executive process, policy guidance and so on. The system model has established connection between individual internal belief, attitude, intention and individual external environmental constraints, controllable interference factors.

Acceptance Model of Human Resource Management System in the Cloud Era. In Davis's technology acceptance model, external variables cover a wide range. The process is black-boxed, after conducting detailing and materialization on the model, modified cloud-computing-based human resource management system acceptance model is proposed. As shown in Figure 1, two conclusive influence factors - the antecedents factors of perceived usefulness and perceived ease of use can be broadly divided into three factor groups: external environment, organizational characteristics, and users 'individual characteristics.

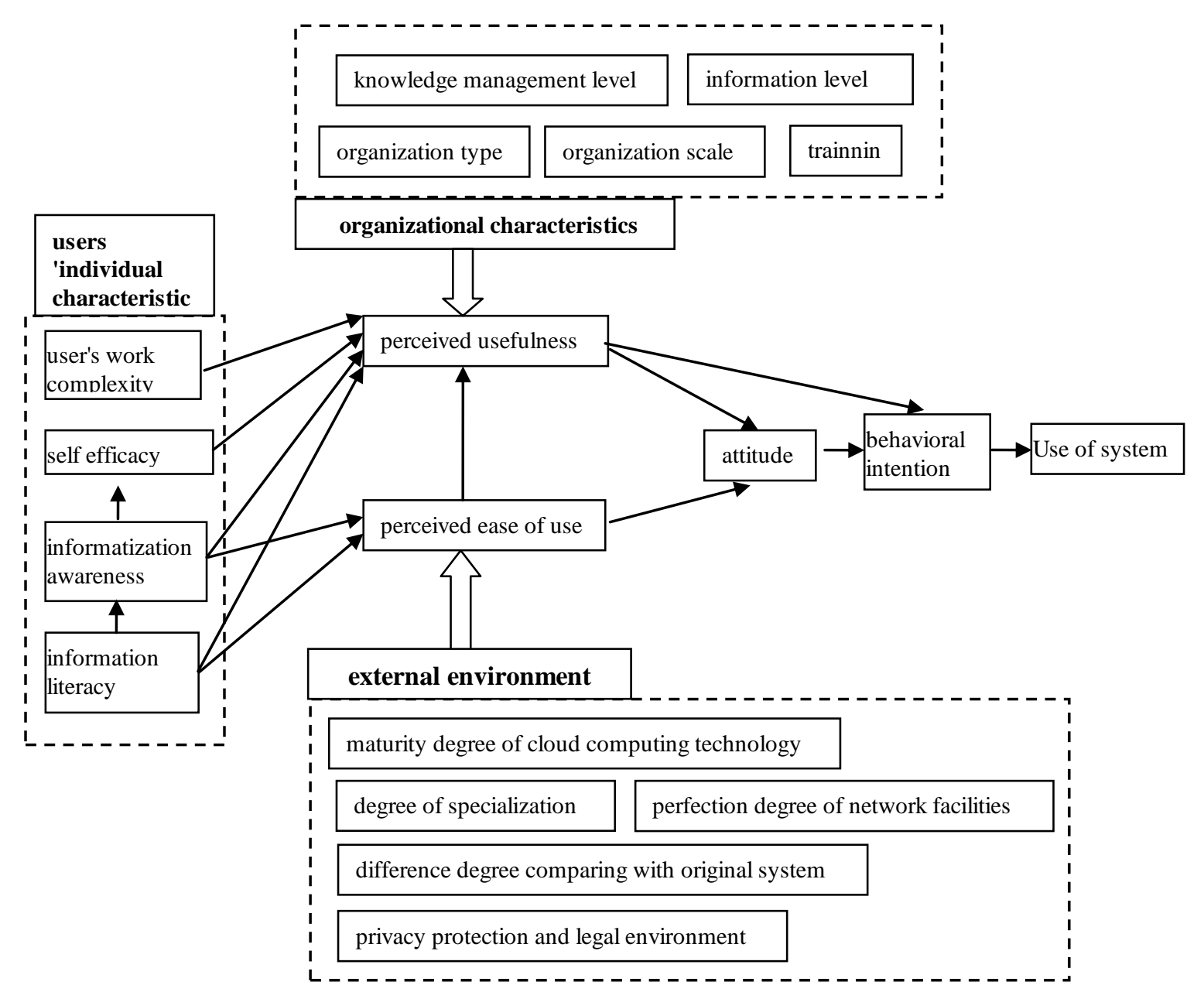

Figure 1. Cloud-computing-based human resource management system acceptance model

Factor Group of External Environment. Factor group of external environment includes maturity degree of cloud computing technology, degree of specialization and difference degree comparing with original system, perfection degree of network facilities, privacy protection and legal environment, the aspects mentioned above will significantly affect the perceived ease of use.

Maturity degree of cloud computing technology. The popularization and application of new technology will be a long process, and new technology will gradually become mature and get improved in this process, as well as conform to users' needs, making users gradually feel the ease of 
use of the new technology. It can be predicted that, with the development and maturity of cloud computing technology, users will gradually perceive ease of use of cloud computing technology, and generate willingness desiring to use

Specialization degree of Cloud computing system. The higher specialization degree of cloud computing system, the more it can meet user's individuation demand, the more it can solve user's specific problems, that is, to achieve "professionalization customization", "customization as required", making users experience ease of use and good use.

Difference degree comparing with existing system. If the information management model, operation interface, process design of cloud computing systems have larger difference from traditional information management system, it will cause user's discomfort. If contrary to the users' habits, it will significantly lower down user's perceived ease of use, therefore, the difference degree from comparing cloud computing system with existing system and user's perceived ease of use is significantly negatively correlated.

Network infrastructure. After migrating cloud computing from enclosed environment to network environment, the data operation and processing are both in distal cloud server. It will significantly influence users' usage experience whether the network is unobstructed or not. Stable and full coverage of network infrastructure will improve the accessibility to accept cloud services, and effectively enhance users' perceived ease of use.

Privacy protection and legal environment. A full range of privacy protection and sound legal protection can reduce the uncertainty during users' usage, and improve user's perceived ease of use.

Factor Group of Organizational Characteristic. Factor group of organizational characteristic includes organization type, organization scale, and the knowledge management level of organization, the information level and relevant training of organization, which will significantly affect the factor perceived usefulness.

Organization type. Comparing with traditional industry organization, sci-tech type organization is more likely to realize the change and efficiency promotion on human resource management by cloud computing, and perceive the usefulness on human resources management by cloud computing technology, furthermore, there will be greater adoption possibility.

Organization scale. Large-scale enterprise organizations have more complex human resource management demand than small and medium organizations, and more urgent desire for new technology. Therefore, cloud computing is more easily to perceive usefulness, expecting that cloud computing can improve the efficiency of human resources management, reduce clerical work, and realize the intellectualization of human resource planning decision-making.

Knowledge management level. The enterprises with a high level of knowledge management knowledge are often learning-driven enterprises, good at knowledge innovation and application, and willing to absorb new technology. Knowledge - based companies are good at discovering reform brought to organization and business environments by new technology, and actively adapt and integrate.

Informatization level. Cloud computing, as a new round of information technology reform, the enterprise organizations with higher informatization degree are more likely and more willing to achieve cloud transformation. Whether from the enterprise's knowledge reserve or technical talent reserves, both are sufficient to cope with the changes brought by new technology, and find the usefulness of new technology.

Training. Through training and technical exchanges and other channels, organizations can from outside know, predict and judge what cloud computing has brought, what problems can be solved for the organizations. After fully understand the characteristics and advantages of cloud computing, organization will perceive its usefulness, and enhance the usage willingness.

Factor Group of Individual Characteristic. Factor group of individual characteristic includes user's work complexity, self efficacy, informatization awareness and information literacy, which can influence the perceived usefulness and perceived ease of use. 
Work complexity. The higher work complexity of the users, the more expected to reduce working strength through using new technologies, the more easily to experience strong booster action on work by information system;

Self efficacy. Self efficacy refers to owned ability judgment, beliefs, self hold and feeling whether an individual can complete one activity on a certain level. Self efficacy can determine the choice of behavior task, as well as the persistence and effort level on this task. Users with a strong sense of self efficacy are more willing to try new ways to solve work problems, and the more can perceive the usefulness of new technology.

Informatization awareness and information literacy. Users with strong informatization awareness and higher information literacy are usually more familiar with information system, easier to adapt the application of new technology, more willing to explore the use skills of new technology, find the advantages of new technology. Therefore, it can remarkably enhance the user's perceived ease of use and perceived usefulness on new technology.

\section{Conclusion}

The above models have only describes the influence of various external factors on the acceptability of cloud-computing-based human resource management system, and assumed positive and negative effects of these factors. Follow-up studies can conduct quantitative study based on this model. Determine the model independent variables, intermediate variable, dependent variable; design the questionnaire; select the sample, carry our data sampling; analyze the suitability degree of model and measure the relation strength degree among various influence factors through structural equation model.

\section{References}

[1] Information on http://www.vsharing.com/k/HR/2002-5/445047.html(In Chinese)

[2] J. Z. Luo, J. H. Jin, A.B. Song, F. Dong: Journal on Communications,32(2011)No.7,p.3-21(In Chinese)

[3] P.C. Du, M. Li, Y. Tong: Human Resources Development of China,(2013)No.15,p.14-18(In Chinese)

[4] Y. Wang: Electronic Test,(2013)No.14,p.271-272(In Chinese)

[5] H.Y. Ma, J.C. Wang: Logistics Engineering and Management,(2014)No.02,p.121-122(In Chinese)

[6] G.L. Zhao: Foreign Economic Relations \& Trade,(2013)No.04,p.127-129(In Chinese)

[7] J.W. Huang: Computer Engineering,(2012)No.24,p.32-36(In Chinese)

[8] X.L. Xuan, C. Zhou: Academic Forum,(2013)No.03,p.159-162+194(In Chinese)

[9] J.P. Ma: CEOCIO China, (2012) No. Z1, p. 58-59 (In Chinese)

[10] Information on http://www.csdn.net/article/2012-04-17/2804667(In Chinese)

[11]F. Davis: MIS Quarte,3(1989)No.13,p.319-341 